Ann. Zootech., I972, 21 (I), I29-I3I.

\title{
EFFET DE LA SIMULTANÉITÉ DE LA LACTATION ET DE LA GESTATION \\ SUR LES PERFORMANCES LAITIERES CHEZ LA LAPINE
}

\author{
F. LEBAS \\ avec la collaboration technique de Marie-Claude Cousin et G. SARDI \\ Station de Recherches sur l'Élevage des Porcs, \\ Centre national de Recherches zootechniques, I. N.R. A., \\ 78 - Jouy-en-Josas
}

La saillie post partum des lapines permet d'accélérer le rythme de reproduction. Différents chercheurs ont étudié l'effet de la lactation sur la fertilité ou la fécondité des lapines (ADAMs, 1967; Prud'hon et BeL, ig68; Smidt et al., I968; Prud'hon et al., i969; Harned et Casida, i969). Nous avons cherché à déterminer, à l'inverse, l'influence de l'état de gestation sur les performances laitières des lapines. (race: Californien).

Nous avons donc comparé les productions laitières de 20 lapines saillies 48 heures après la mise bas à celles de 20 contemporaines non gestantes. Les 40 femelles en expérience ont été utilisées au cours de leur $2^{\mathbf{e}}$ lactation. Sur les 20 lapines saillies post partum, 12 seulement se sont révélées gestantes. Ces dernières ont donc été seules prises en considération. Les conditions d'élevage, d'alimentation et de contrôle de la production laitière ont été semblables à celles que nous avons décrites dans un travail précédent (LEBAS, 1968) ; toutefois, la production laitière a été contrôlée

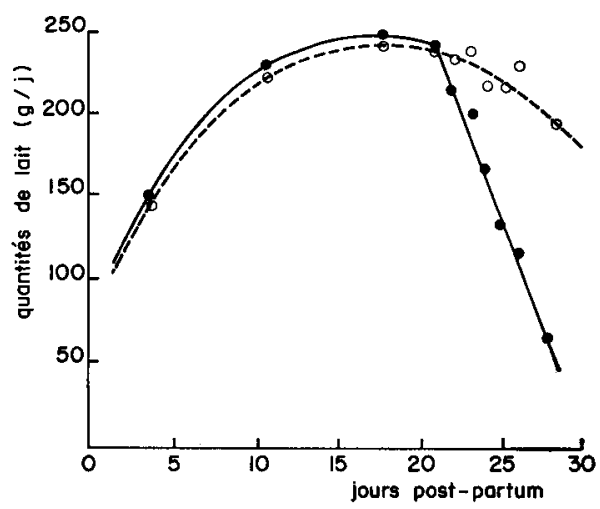

FIG. r. - Évolution de la production laitière de lapines simultanément gestantes et allaitantes $(\bullet-\bullet)$ ou simplement allaitantes $\left(0_{-}-\mathrm{O}\right)$ 
6 jours sur 7 et non I jour sur 2 pour assurer une meilleure précision. Pour l'ensemble des lapines, la lactation a été arrêtée par sevrage des lapereaux à 28 jours après la mise bas.

Pour les différentes lapines, le nombre de lapereaux allaités était situé entre 7 et ro. L'effectif de portée moyen a été semblable pour les 2 traitements : 8,5 $\pm 0,25$. Les performances laitières sont réunies sur la figure r. Avant le $22^{\mathbf{e}}$ jour, il n'est possible de déceler aucune différence significative entre les 2 catégories de lapines; par contre, après le $22^{\mathbf{e}}$ jour, les lapines gestantes ont une production laitière qui décroît beaucoup plus rapidement que celle des lapines témoins. Cette production plus faible va de pair avec une consommation réduite d'aliment concentré durant la $4^{\text {e }}$ semaine (tabl. I). Au cours de cette période, comme des précédentes d'ailleurs, nous n'avons observé aucune différence significative dans l'évolution du poids vif des lapines (tabl, 2). Les performances des lapereaux, résumées au tableau 3, ont également été semblables pour les deux traitements.

\section{TABIEAU I}

Consommation d'aliment granulé par les lapines allaitantes nourries à volonté (en g)

\begin{tabular}{|c|c|c|c|c|c|}
\hline Semaines & 1 & 2 & 3 & $4_{4}$ & Total \\
\hline Lapines gestantes $\ldots$. & $2610 \pm 70$ & $2980 \pm 50$ & $2920 \pm 90$ & $2250 \pm 100$ & $10770 \pm 230$ \\
\hline Lapines témoins $\ldots \ldots$ & $2540 \pm 110$ & $2780 \pm 140$ & $2980 \pm 90$ & $2870 \pm 130$ & $11160 \pm 360$ \\
\hline Signification $\ldots \ldots \ldots$ & NS & NS & NS & $\mathrm{P}<0,05$ & NS \\
\hline
\end{tabular}

TABLEAU 2

Évolution du poids vif des lapines allaitantes (en $\mathrm{kg}$ )

\begin{tabular}{|c|c|c|c|c|c|}
\hline Jours Post-partum & 1 & 7 & 14 & 21 & 28 \\
\hline Lapines gestantes ... & $4,93 \pm 0,10$ & $4,36 \pm 0,09$ & $4,53 \pm 0,09$ & $4,6^{\prime} \pm 0,09$ & $4,52 \pm 0,10$ \\
\hline Lapines témoins .... & $4,16 \pm 0,08$ & $4,4_{4}^{2} \pm 0,08$ & $4,47 \pm 0,10$ & $4,54 \pm 0,10$ & $4,49 \pm 0,09$ \\
\hline Signi fication . . . . . . . & NS & NS & NS & NS & NS \\
\hline
\end{tabular}

TABLEAU 3

Performances moyennes des portées de lapereaux

\begin{tabular}{|c|c|c|c|c|}
\hline & \multicolumn{2}{|c|}{$\begin{array}{l}\text { Poids de portée } \\
\qquad(g)\end{array}$} & \multirow{2}{*}{$\begin{array}{l}\text { Consommation d'aliment } \\
\text { concentré par portée } \\
\text { jusqu'au sevrage }(g)\end{array}$} & \multirow{2}{*}{ Effectif sevré } \\
\hline & $21 \mathrm{j}$ & $28 \mathrm{j}$ & & \\
\hline Mères gestantes .. & $2750 \pm 90$ & $4560 \pm 130$ & $1510 \pm 100$ & $8,42 \pm 0,31$ \\
\hline Témoins........ & $2790 \pm 110$ & $46,6 \pm 180$ & $1480 \pm 120$ & $8,25 \pm 0,24$ \\
\hline
\end{tabular}


La simultanéité de la gestation et de la lactation n'affecte donc pas la production laitière durant les 3 premières semaines. Par contre, à partir du $22^{\mathbf{e}}$ jour, on enregistre une diminution pratiquement linéaire de la production laitière. A ce stade, le lait ne représente qu'une faible partie de l'alimentation des lapereaux (LeBAs, I970 a) ; il est donc normal de ne constater aucune différence dans les performances des portées, ni pour la consommation d'aliment concentré, ni pour la croissance pondérale. La réduction de l'ingestion en $4^{\mathrm{e}}$ semaine pour les lapines gestantesallaitantes correspond exactement à celle observée chez les lapines simplement gestantes nourries à volonté à partir du $20^{\mathrm{e}}$ jour de gestation (LeBAs, I970 b). La baisse de la production laitière pourrait alors s'expliquer par un manque de nutriments pour la synthèse du lait, compte tenu d'une priorité accordée dès lors à la gestation. C'est, en effet, à partir de ce stade que la croissance des embryons s'accélère et représente un dépôt de matières appréciable chaque jour. La stabilité du poids vif des lapines gestantes par rapport aux témoins est à notre sens une preuve de cette compétition. En tout état de cause, l'élaboration d'une nouvelle portée par la mère durant l'allaitement de la précédente ne porte aucun préjudice à cette dernière.

Reçu pour publication en septembre 1971.

\section{SUMMARY}

\section{EFFECT OF CONCURRENT LACTATION AND PREGNANCY ON THE MILK PERFORMANCES OF THE RABBIT}

Lactation performances of $\mathrm{r} 2$ concurrently suckling and pregnant does were compared with those of 20 contemporaries. The matings were performed 48 hours post partum. The milk productions were similar until the 2 ist day included. From the 22 nd day after parturition, the milk yield of the pregnant does decreased very rapidly (fig. I). Compared with the controls, the weight of the does and the performances of the litters were not modified (tables 2 and 3 ). On the other hand, the intake of concentrate food by the pregnant females was lower during the $4^{\text {th }}$ week. The decrease in milk production could be due to a competition between the syntheses of the embryo and the mammary gland. The fact that there was no variation in the weight of the pregnant does in comparison with the controls, supports this hypothesis (table I).

\section{RÉFÉRENCES BIBLIOGRAPHIQUES}

Adams C. E., 1967. Concurrent lactation and pregnancy in the Rabbit. J. Reprod. Fertil., 14, 35I-352. Harned M. A., Casida L. E., I969. Some post partum reproductive phenomena in the domestic rabbit. J. Anim. Sci., 28, 785-788.

Lebas F., I968. Mesure quantitative de la production laitière chez la lapine. Ann. Zootech., 17, I69-I82.

Lebas F., I970 $a$. Alimentation et croissance du lapereau sous la mère. Rec. Méd. vét. (Alfort), 146, I065-1070.

Lebas F., I970 $b$ (Données non publiées).

Prud'hon M., Bel L., x968. Le sevrage précoce des lapereaux et la reproduction des lapines. Ann. Zootech., 17, 23-30.

Prud'hon M., Rouvier R., Cael J., Bel L,, I969. Influence de l'intervalle entre la parturition et la saillie sur la fertilité et la prolificité des lapines. Ann. Zootech., 18, 3I7-329.

Smidt D., Thоmsen J. J., Hafez E. S. E., ig68. Untersuchungen zum Einfluss der Laktation auf Implantation und Fötalentwicklung beim Kaninchen. Z. Tierzucht. Zucht. Biol., 84, I I5-I 26. 